\title{
Supporting Multipoint-to-Point Communications in All-Optical WDM Networks
}

\author{
Fen Zhou \\ IRISA / INSA Rennes \\ Campus de Beaulieu \\ Rennes, France, 35042 \\ Email: fen.zhou@irisa.fr
}

\author{
Mohand Yazid Saidi \\ IRISA / University of Rennes 1 \\ Campus de Beaulieu \\ Rennes, France, 35042 \\ Email: msaidi@irisa.fr
}

\author{
Miklós Molnár \\ IRISA / INSA Rennes \\ Campus de Beaulieu \\ Rennes, France, 35042 \\ Email: molnar@irisa.fr
}

\author{
Bernard Cousin \\ IRISA / University of Rennes 1 \\ Campus de Beaulieu \\ Rennes, France, 35042 \\ Email: bernard.cousin@irisa.fr
}

\begin{abstract}
The routing and wavelength assignment (RWA) problem for multipoint-to-point communications in all-optical wavelength division multiplexing (WDM) networks is investigated in this paper. Two efficient algorithms, namely Reverse Shortest Path Tree routing (RSPT) and $k$-Bounded Edge Disjoint Path routing (EDPR), are proposed. The problem of minimizing the total cost while establishing a multipoint-to-point session can be solved by RSPT algorithm in polynomial time. Nevertheless, EDPR algorithm produces a significant reduction in the maximum number of wavelengths required per link (i.e., the link stress) by a multipoint-to-point session. Simulations demonstrate the efficiencies of these two algorithms in supporting multipointto-point communications in WDM networks.
\end{abstract}

Index Terms-All-optical WDM Networks, Multipoint-to-point Communications, Routing and Wavelength Assignment (RWA), Light-startree

\section{INTRODUCTION}

All-optical Wavelength Division Multiplexing (WDM) networks, which are built on the concept of wavelength routing, have been deployed in the Internet infrastructure for decades to satisfy increasing demand for bandwidth [1]. As optical crossconnects (OXCs) become mature and commercially available, it enables a light beam to pass an intermediate optical node without the optical-electrical-optical (OEO) conversion. By widely employing optical OXCs, all-optical WDM networks overcome the electro-optic bottleneck and thus are gifted at providing huge bandwidth, low signal attenuation and distortion as well as favorable transmission delay [2]. Relying on their inherent advantages, WDM networks are capable of supporting bandwidth-driven multimedia services in the Internet, such as VoIP, video conference, online communitybased communications (e.g., blog, voting), etc. It broadly consists of four traffic patterns: point-to-point communications (or unicast), point-to-multipoint communications (multicast), multipoint-to-point communications (MP2P) and multipointto-multipoint (MP2MP) communications.

To support unicast communications in all-optical WDM networks, lightpaths are introduced to reduce the number of hops that a packet has to traverse [3]. A lightpath is a set of consecutive wavelength channels, which connects a transmitter at a source node to a receiver at a destination node.

To satisfy the multicast services in all-optical WDM networks, the lightpath is extended to a light-tree [3] to minimize the number of transceivers and the average hop distance for given traffic demands. A light-tree can be viewed as a point-tomultipoint generalization of a lightpath. But, the network node should be equipped with optical power splitters to construct the multicast light-trees. In the case of sparse light splitting, where only a small fraction of network nodes are capable of splitting, the light-forest [4] concept is introduced.

For multipoint-to-multipoint case, the core-based tree (CBT, [5]) structure could be applied with some proper modifications. In a core-based tree structure, a core node is employed to collect messages from all the source nodes and then forward the collected messages to the destination nodes.

Supporting multipoint-to-point communications is also important and indispensable for wavelength-routed WDM networks. To the best of our knowledge, no literature has addressed this problem before. Therefore, in this paper, we investigate the routing and wavelength assignment (RWA) problem for multipoint-to-point communications in all-optical WDM networks. The following assumptions are made in our study (i) the number of wavelengths supported per fiber link is sufficient to establish a multipoint-to-point communication, (ii) wavelength converters are not available due to the high cost and complex architecture, (iii) the optical traffic grooming switches [6], which enable to aggregate low speed traffic streams into one high capacity wavelength, are also unavailable because of the lack of intelligent network control and automatic provisioning functionality [7].

An optical multipoint-to-point communication generally involves a simultaneous light signal transmission from several source nodes to the same sink node. In all-optical WDM networks, two basic physical constraints are always encountered. The first one, namely wavelength continuity constraint, implies the same wavelength should be retained along a lightpath without wavelength conversion. And the other one, called the distinct wavelength constraint, does not permit to assign the same wavelength for two lightpaths sharing a common fiber link. For multipoint-to-point communications in all-optical networks, the distinct wavelength constraint is even more strict. The light signals emitted by two source nodes can be carried on the same wavelength only if the lightpaths from these two source nodes to the same sink node are edge disjoint. Thereby, we introduce a light-startree structure to 


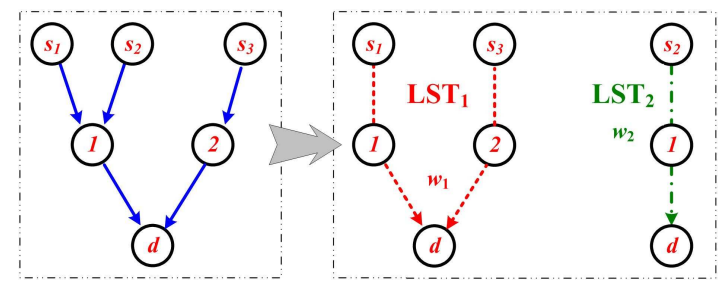

Fig. 1. An example of light-startrees

route a multipoint-to-point communication. A light-startree is consisted of several edge disjoint lightpaths terminated at the same sink node but assigned only one wavelength. An example of light-startrees is given in Fig. 1. In the left dash-dotted rectangle, a multipoint-to-point communication demande $\operatorname{mp} 2 p\left(\left(s_{1}, s_{2}, s_{3}\right), d\right)$ arrives. Two light-startrees in the right dash-dot rectangle (i.e., wavelength $w_{1}$ is assigned to $L S T_{1}$, and $w_{2}$ to $L S T_{2}$ ) can be used to route this session.

Based on the light-startree structure, two efficient routing and wavelength assignment (RWA) algorithms are presented in our paper. The proposed Reverse Shortest Path Tree routing algorithm is able to minimize the wavelength channel cost (i.e., the total cost). To reduce the maximum number of wavelengths required per link (i.e., link stress) by a multipointto-point communication request, a $k$-Bounded Edge Disjoint Path routing algorithm is introduced. Extensive simulations are done to assess the performance of these two algorithms.

The rest of this paper is organized as follows. Section II formulates the RWA problem for multipoint-to-point communications in WDM networks. Two RWA algorithms are introduced in Section III. Numerical results are obtained in Section IV. Finally, a summary of this paper is made in Section V.

\section{Multipoint-To-POint \\ COMmunications in ALL-Optical WDM Networks}

In this article, the routing and wavelength assignment (RWA) problem for multipoint-to-point communications in alloptical WDM networks is studied. A WDM network can be modeled by an undirected graph $G(V, E, c, W)$. $V$ denotes the set of nodes in the network. Each edge $e \in E$ is associated with a weight function $c(e)$, which signifies the cost of fiber link channel e. $c(e)$ is additive along a lightpath $L P(u, v)$, where $u$ and $v$ are two end point nodes of the lightpath. $W$ denotes the number of wavelengths that could be supported in a fiber link. We consider a multipoint-to-point session $m p 2 p(S, d)$, which requires to set up simultaneous lightpaths from each source $s_{i} \in S$ to the same destination $d$. Due to the distinct wavelength constraint in WDM networks, two lightpaths could be assigned the same wavelength only if they are edge disjoint. Hence, the multipoint-to-point communication can be realized either by an individual unicast path to the destination for each source or a set of light-startrees. A lightstartree is a set of edge-disjoint lightpaths, which share the same receiver end point and occupy the same wavelength. One light-startree may not be sufficient to span all the source nodes of a multipoint-to-point session, and thus several lightstartrees may be used with each on a different wavelength. To carry out a multipoint-to-point session, a RWA algorithm should try to save as many wavelength channel cost as possible while overcoming the optical constraints. The total cost of a multipoint-to-point communication can be calculated by the sum of each fiber link channel cost in the set of light-startrees ( $L S T)$ computed out. i.e.,

$$
c[m p 2 p(S, d)]=\sum_{L S T_{i} \in m p 2 p(S, d)} \sum_{e \in L S T_{i}} c(e)
$$

Besides this, the maximum number of wavelengths required per link for a multipoint-to-point session (i.e., link stress) is also important for WDM networks. This is because, physically, the number of wavelengths supported on a fiber link is limited to a fixed number. If a multipoint-to-point session spends too many wavelengths in one fiber link, then the number of wavelengths left for other sessions will be limited, which thus results in the blocking of some sessions. Hence, balancing the multipoint-to-point traffic and avoiding the blocking should also be taken into account by an efficient routing algorithm. Generally, the set of light-startrees computed out for a multipoint-to-point session are not edge disjoint and thus the link stress equals the number of light-startrees and at most the number of source nodes in a multipoint-to-point session.

\section{Proposed Solutions}

In this section, two RWA algorithms are proposed for multipoint-to-point communications in all-optical WDM networks. The first one, namely Reverse Shortest Path Tree routing algorithm, tries to optimize the total cost for a session. The second one, namely $k$-Bounded Edge Disjoint Path Routing algorithm (EDPR), is primarily designated to minimize the link stress for a multipoint-to-point session. This algorithm balances the traffic load on the network fiber links. Apart from the primary objective, algorithm EDPR also tries to optimize the total cost as the second objective.

\section{A. Reverse Shortest Path Tree Routing Algorithm (RSPT)}

Algorithm RSPT solves the routing and wavelength assignment problem for a multipoint-to-point session separately. The shortest path tree SPT rooted at destination $d$ is first constructed. Then, the edges in the shortest path tree SPT are reversed in order to transmit light signals from each source $s \in$ $S$ to destination $d$. After the search of routes, the wavelength assignment is implemented to satisfy the distinct wavelength constraint. Different wavelengths have to be employed for any pair of source nodes if their shortest paths to $d$ share at least a common link. In the absence of optical traffic grooming switches [7], algorithm RSPT is able to provide the optimal total cost for a MP2P session. In addition, it can be solved in the time $O(|V| \log |V|+|E|)$ by Dijkstra's algorithm.

\section{B. k-Bounded Edge Disjoint Path Routing Algorithm (EDPR)}

Although algorithm RSPT optimizes the total cost, it is not competitive for minimizing the link stress. This can 


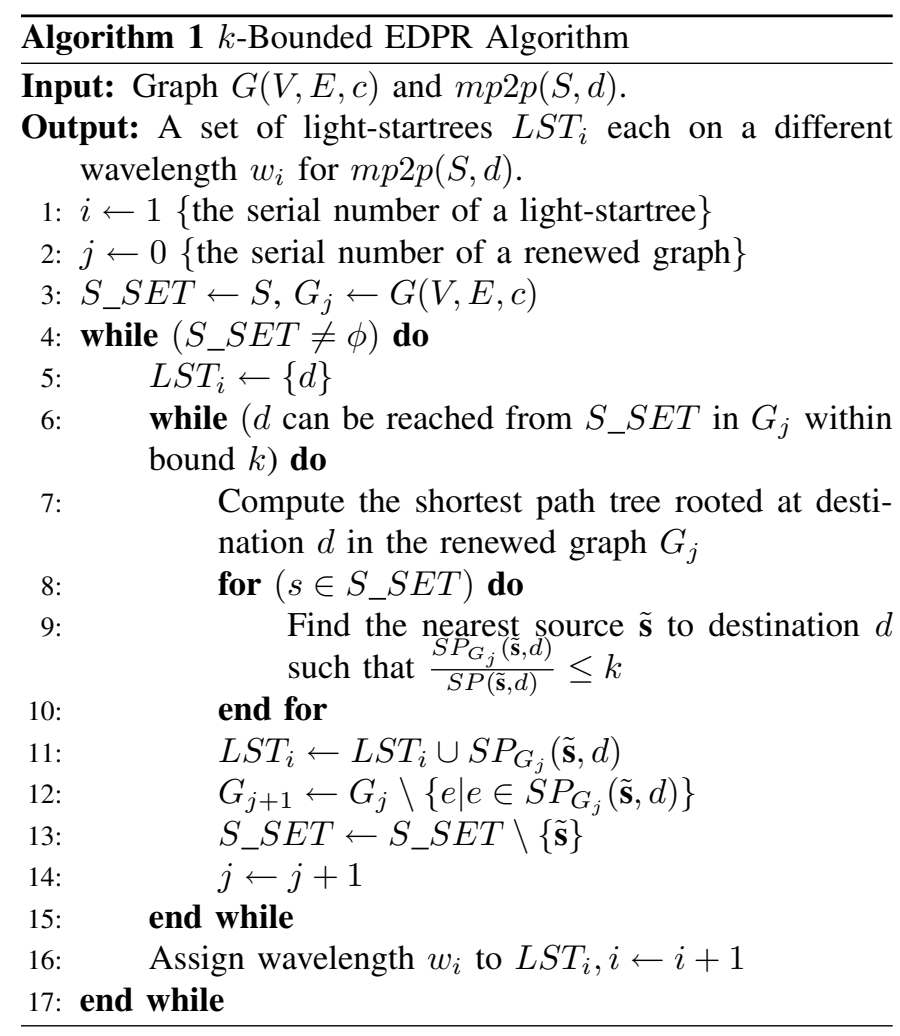

be explained as follows. Algorithm RSPT only routes the multipoint-to-point traffic over the shortest paths, but it does not consider the relevant relation between the paths from the sources to the destination. Especially, some fiber links around the destination and leading to source nodes will be frequently used. Since different light signals are transmitted over each shortest path, the fiber links commonly shared by these shortest paths are highly loaded in the absence of costly optical traffic grooming switches [7]. The more traffic is concentrated on a fiber link, the more wavelengths will be required. Although sometimes we can not avoid the frequent usage of the links adjacent to the destination, we can at least balance the traffic among these fiber links. As the traffic load is balanced and evenly distributed, the maximum number of wavelengths needed per fiber link (i.e., link stress) is reduced. Based on this fact, a $k$-Bounded Edge Disjoint Path Routing algorithm is proposed.

Different from the RSPT algorithm, the $k$-Bounded Edge Disjoint Path Routing algorithm considers the relationship between the lightpaths from all sources to the destination while searching the routing paths. It aims to find the maximum edge disjoint paths for the source nodes. To achieve this, an edge removal action is introduced during the lightpath computation. The edge removal action takes place whenever the lightpath from a source node to destination $d$ is found. At the $j^{\text {th }}$ step, the source $\tilde{\mathbf{s}}$ nearest to destination $d$ in the renewed graph $G_{j}$ is added to light-startree via the shortest path $S P_{G_{j}}(\tilde{\mathbf{s}}, d)$ such that it holds $\frac{S P_{G_{j}}(\tilde{\mathbf{s}}, d)}{S P(\tilde{\mathbf{s}}, d)} \leq k$. To avoid using the same link twice, all the links in the lightpath specified for a source will be removed from the graph (refer to step-12). Then, the nearest source is computed in the renewed graph and the shortest path in the renewed graph is specified as the lightpath for the nearest destination. It is true that, most of the time, one lightstartree is not sufficient to span all the sources. In case that the destination is not reachable from the unspanned sources in the renewed graph, another light-startree assigned a new wavelength will be computed over the initial topology using the same technique.

\section{NumericAl Results}

Numerical results are obtained by the simulations. The USA Longhaul topology, which is viewed as a good candidate for the future backbone network of USA, is selected as the WDM network testbed. Destination and source nodes are assumed to be distributed uniformly and independently in the topology for each multipoint-to-point session. An equal cost of 1 unit hopcount cost is assumed on all the links to simplify the simulation. Two metrics are considered to evaluate the quality of the computed light-startrees for $m p 2 p(S, d)$ :

- Total Cost: wavelength channels cost in the light-startrees computed for $m p 2 p(S, d)$. It is calculated by equation (1).

- Link Stress: the maximum number of wavelengths required per link to span all the source nodes in $m p 2 p(S, d)$.

\section{A. Impact of Bound $k$ in EDPR Algorithm}

The bound $k$ should be chosen carefully, since it has a great impact on the performance of the algorithm. Here, we study the quality of light-startrees when the bound $k$ varies, which could be a good reference for the selection of bound $k$.

In Fig. 2, the cost approximation ratio of EDPR algorithm is plotted when the session members ratio corresponds to $10 \%$, $50 \%$ and $100 \%$ respectively. Since the cost of RSPT is optimal, the cost approximation ratio is computed by $\frac{c(E D P R)}{c(R S P T)}$. As indicated, the approximation ratio becomes worse as the the bound $k$ grows. We can also see that, the approximation ratio stays stable when $k$ increases beyond a certain value. Because the diameter of a network is a fixed value (e.g., USA Longhaul Network has a diameter of 8 ), the value of $\frac{S P_{G_{j}}(\tilde{\mathbf{s}}, d)}{S P(\tilde{\mathbf{s}}, d)}$ is limited. Once $k$ increases big enough, EDPR algorithm can get the same total cost as that when $k$ is infinite.

The tendency of link stress versus the bound $k$ is shown in Fig. 3. The link stress of EDPR algorithm diminishes as the bound $k$ grows. The EDPR algorithm can reach the best link stress when $k=1.8$. After that value, the link stress keeps steady. This can be interpreted as follows. As $k$ increases, the condition of the possible candidate lightpaths from a source to destination $d$ is relaxed. Then more possible lightpaths can be found for a source. However, similar to the total cost, there will be no longer any constraint on the length of a possible lightpath if $k$ is large enough. Consequently, link stress will be stable. 


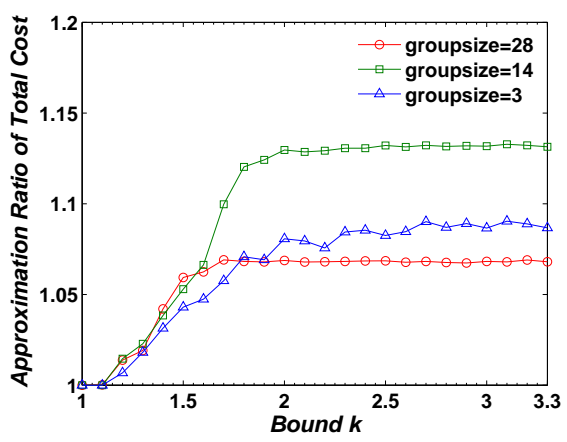

Fig. 2. Approximation of Total Cost versus Bound $k$

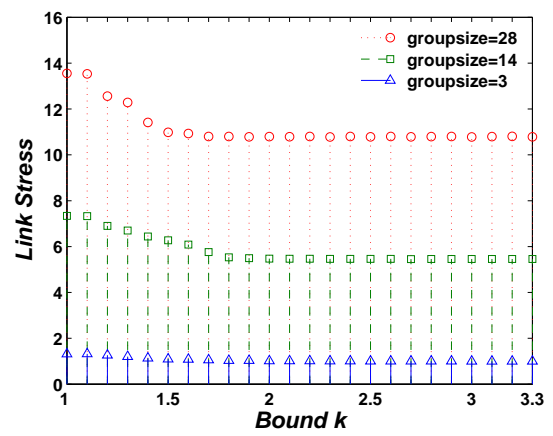

Fig. 3. Link Stress versus Bound $k$

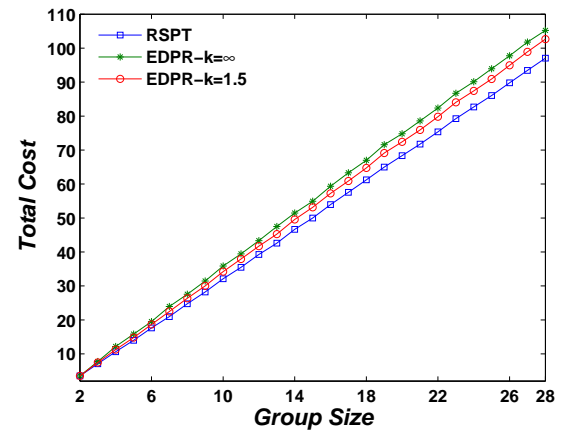

Fig. 4. Total Cost against Multipoint-to-point Session Group Size

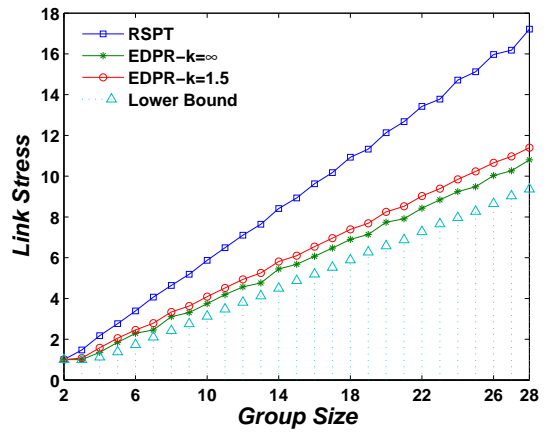

Fig. 5. Link Stress against Multipoint-to-point Session Group Size

\section{B. Comparison of RSPT and k-Bounded EDPR}

In Figs. 4 and 5, the quality of light-startrees computed by RSPT and EDPR algorithms are compared. The lower bound of link stress plotted in Fig. 5 is calculated by $\max \left\{\left\lceil\frac{|S|}{\operatorname{Deg}(d)}\right\rceil, 1\right\}$. Based on the simulation results, both the total cost and the link stress race up rapidly when the MP2P session group size increases. The RSPT algorithm is able to get the best total cost, while EDPR algorithm with $k=\infty$ results in the best link stress which is very close to the lower bound. When $k=1.5$, the total cost of EDPR algorithm is better than that of $k=\infty$, while its link stress is less favorable than that of $k=\infty$. So, a tradeoff should be found between the total cost and the link stress to satisfy different types of multimedia multipoint-to-point traffics.

\section{CONCLUSION}

Routing and wavelength assignment is a very important issue for supporting multipoint-to-point communications in all-optical WDM networks. Distinct wavelengths should be employed by source nodes to send light signals to the same destination $d$, if their lightpaths leading to $d$ are not edge disjoint. The light-startree structure is introduced to route multipoint-to-point communications, which consists of several edge disjoint lightpaths assigned the same wavelength and terminated at the same destination. To establish a multipointto-point session, a set of light-startrees, each with a different wavelength, may be required. As the number of wavelengths supported in one fiber link is limited, not only the total cost but also the link stress are important parameters to measure the quality of light-startrees computed. For this reason, the Reverse Shortest Path Tree routing algorithm is proposed, which is able to to minimize the total cost in polynomial time. Meanwhile, the $k$-Bounded Edge Disjoint Path Routing algorithm is presented to route a multipoint-to-point session with as fewer wavelengths as possible. It results in significant reduction of the link stress. Numerical results obtained demonstrate the efficiency of the proposed algorithms in supporting all-optical multipoint-to-point communications.

\section{REFERENCES}

[1] George N. Rouskas. Optical layer multicast: rationale, building blocks, and challenges. IEEE Network, 17(1): 60-65, 2003.

[2] Biswanath Mukherjee. WDM Optical communication networks: progress and challenges. IEEE Journal on Selected Aeras in Communications, 18(10): 1810-1824, 2000.

[3] Laxman H. Sahasrabuddhe, Biswanath Mukherjee. Light-trees: optical multicasting for improved performance in wavelength-routed networks. IEEE Communications Magazine, 37(2): 67-73, 1999.

[4] Xijun Zhang, John Wei, Chunming Qiao. Constrained multicast routing in WDM networks with sparse light splitting. IEEE/OSA Journal of Lightware Technology, 18(12): 1917-1927, 2000.

[5] Jianping Wang, Xiangtong Qi, Mei Yang. Routing and wavelength assignment for core-based tree in WDM networks. Computer Communications, 29: 1896-1904, 2006.

[6] Osama Awwad, Ala I. Al-Fuqaha, Ammar Rayes. Traffic grooming, routing, and wavelength assignment in WDM transport networks with sparse grooming resources. Computer Communications, 30: 3508-3524, 2007.

[7] Keyao Zhu, Hui Zang, Biswanath Mukherjee. A comprehensive study on next-generation optical grooming switches. IEEE Journal on Selected Areas in Communications, 21(7): 1173-1186, 2003. 